\title{
Atividade anti-helmíntica dos flavonóides isolados das raízes de Andira anthelmia (Leguminosae)
}

\author{
Virgínia C. da Silva, ${ }^{1}$ Mário G. de Carvalho, ${ }^{*, 1}$ Hélcio R. Borba, ${ }^{2}$ Sandra L. C. Silva ${ }^{3}$ \\ ${ }^{1}$ Instituto de Ciências Exatas, Universidade Federal Rural do Rio de Janeiro, Km 47 BR 465, 23890-000 \\ Seropédica-RJ, Brasil, \\ ${ }^{2}$ Instituto de Biologia, Universidade Federal Rural do Rio de Janeiro, Km 47 BR 465, 23890-000 \\ Seropédica-RJ, Brasil, \\ ${ }^{3}$ Departamento de Estudos Básicos e Instrumentais, Universidade Federal do Sudoeste da Bahia, Campus de \\ Itapetinga, Km 3 BR 415, 45700-000 Itapetinga-BA, Brasil
}

\begin{abstract}
RESUMO: As cascas e as raízes de $A$. anthelmia têm sido utilizadas tradicionalmente como vermífugas. Um estudo biomonitorado do extrato metanólico das raízes de $A$. anthelmia conduziu ao isolamento das isoflavonas biochanina A e genisteína da fração acetato de etila; biochanina A 7-O- $\beta$-D-glicopiranosídeo, biochanina A 7- $O$ - $\alpha$-L-rhamnopiranosil- $(1 \rightarrow 6)-\beta$-D-glicopiranosídeo e biochanina A 7-O- $\beta$-D-apiofuranosil- $(1 \rightarrow 5)-\beta$-D-apiofuranosil- $(1 \rightarrow 6)-\beta-\mathrm{D}$-glicopiranosídeo da fração $n$-butanólica e catequina da fração metanólica. Suas estruturas foram elucidadas com base em dados espectrométricos. A atividade anti-helmíntica foi investigada em camundongos naturalmente infectados por Aspiculuris tetraptera. Os compostos isolados aplicados nos animais por via intragástrica na dose de $2,0 \mathrm{mg} . \mathrm{kg}^{-1}$ durante três dias consecutivos revelaram percentuais significativos na eliminação de $A$. tetraptera, quando comparados com o lote controle.
\end{abstract}

Unitermos: Andira anthelmia, flavonóides, atividade anti-helmíntica.

\begin{abstract}
Anthelmintic activity of flavonoids isolated from roots of Andira anthelmia (Leguminosae)". Bioactivity-guided fractions of the methanol extract from the roots of Andira anthelmia led the isolation of the isoflavones biochanin A and genistein from the ethyl acetate fraction; biochanin A 7-O- $\beta$-D-glucopyranoside, biochanin A 7- $O$ - $\alpha$-L-rhamnopyranosyl- $(1 \rightarrow 6)$ $\beta$-D-glucopyranoside and biochanin A 7-O- $\beta$-D-apiofuranosyl- $(1 \rightarrow 5)$ - $\beta$-D-apiofuranosyl- $(1 \rightarrow 6)$ $\beta$-D-glucopyranoside from the $n$-butanol fraction and catechin from the methanol one. Their structures were elucidated on the basis of spectroscopy data. The anthelmintic activity was investigated in mice naturally infected by Aspiculuris tetraptera. The compounds administered in the animals by intragastric route in doses of $2.0 \mathrm{mg} \cdot \mathrm{kg}^{-1}$, were effective in the removal of the total number of the $A$. tetraptera when compared with the control group.
\end{abstract}

Keywords: Andira anthelmia, flavonoids, anthelmintic activity.

\section{INTRODUÇÃO}

O gênero Andira compreende o grupo de plantas popularmente conhecidos por angelins, representado por mais de 30 espécies distribuídas na América Tropical, com uma espécie na África (Matos, 1979; Pennigton \& Lima, 1995), sendo a maioria originária do Brasil (Matos, 1979). No Brasil, foram encontradas 27 espécies e 7 variedades, sendo que o maior número de espécies se encontra na Amazônia (Matos, 1979). Devido às propriedades vermífugas, esse gênero foi utilizado na Europa desde 1755, por médicos e farmacêuticos de diversos países que preconizavam a industrialização das cascas, transformando-as em pó, com o qual procuravam obter uma droga de aplicação anti-helmíntica (Matos, 1979). Algumas espécies que pertencem ao gênero Andira ainda são utilizadas popularmente como anti- helmínticas, apesar de seus efeitos tóxicos (Corrêa, 1984; Silva et al., 2003). Em trabalho anterior divulgamos a avaliação da ação anti-helmíntica dos extratos brutos de $A$. anthelmia e $A$. fraxinifolia, sugerindo o extrato bruto da espécie $A$. anthelmia como um potente anti-helmíntico (Silva et al., 2003). Há facilidade de disseminação de A. tetraptera em grandes criações de roedores, sendo freqüentemente encontrados parasitando o intestino de camundongos mantidos em biotério (Zenner \& Regnault, 2000). O presente estudo procurou investigar o efeito anti-helmíntico das frações geradas pelo fracionamento do extrato metanólico assim como dos compostos isolados das frações cujos resultados foram positivos frente ao biomonitoramento utilizando como modelo experimental camundongos naturalmente infectados com o nematóide Aspiculuris tetraptera. 


\section{MATERIAL E MÉTODOS}

\section{Procedimentos gerais}

Os espectros do $\mathrm{RMN},{ }^{1} \mathrm{H}$ e ${ }^{13} \mathrm{C}$ (inclusive experimentos em 2D) foram registrados em espectrômetros Bruker DRX-500 ( ${ }^{1} \mathrm{H} 500$ e ${ }^{13} \mathrm{C} 125$ $\mathrm{MHz})$; Bruker AS-200 $\left({ }^{1} \mathrm{H} 200\right.$ e $\left.{ }^{13} \mathrm{C} 50 \mathrm{MHz}\right)$ e Jeol JNM-GX-400 ( ${ }^{1} \mathrm{H} 400$ e $\left.{ }^{13} \mathrm{C} 100 \mathrm{MHz}\right)$. O solvente usado na dissolução das amostras foi DMSO-d ${ }_{6}$ e TMS como referência interna. Nas cromatografias em coluna (CC) foram usadas sílica gel (Merck e Aldrich) ou Sephadex LH-20 (Aldrich); nas análises em camada fina utilizouse cromatofolhas de sílica gel $60 \mathrm{PF}_{254}$ (Merck e Aldrich) e revelação com UV (254 e 366 nm), $\mathrm{AlCl}_{3}$-EtOH (1\%) e exibição em vapores de iodo.

\section{Material vegetal}

Raízes de Andira anthelmia foram coletadas em Dezembro de 2001 na Reserva Biológica de Poço das Antas, Município de Silva Jardim (Rio de Janeiro). Uma exsicata ( $\mathrm{n}$ - 4616) encontra-se catalogada no Herbário RBR-UFRRJ.

\section{Extração e isolamento}

Após secageme trituração, 450,0 g deraízes de Andira anthelmia foram submetidas à maceração contínua com metanol à temperatura ambiente. $\mathrm{O}$ extrato metanólico $(76,0 \mathrm{~g})$ foi submetido à partição com solventes em polaridades crescentes obtendo os resíduos com acetato de etila $(19,0 \mathrm{~g})$, butanol $(15,0 \mathrm{~g})$ e metanol $(24,0 \mathrm{~g})$. O resíduo acetato de etila foi cromatografado em coluna de sílica gel e eluída com clorofórmio, acetato de etila e metanol, com aumento de polaridade até $100 \%$ metanol. Foram coletadas 156 frações de $15 \mathrm{~mL}$ que foram analisadas por CCDA e reunidas em 16 novas frações. As frações $6 / 10$ forneceram $1(260,0 \mathrm{mg})$ e $2(28.0 \mathrm{mg})$. A fração butanólica foi submetida à cromatografia em coluna de sílica gel eluída inicialmente com acetato de etila e aumento gradual de metanol. Foram coletadas 185 frações de $15 \mathrm{~mL}$, que novamente por CCDA foram analisadas e reunidas em 25 novas frações. As frações 7/16 foram filtradas em Sephadex LH-20 eluída com methanol fornecendo o composto $3(65,0 \mathrm{mg})$. As frações 17/25 foram submetidas a CCDP eluída com $\mathrm{CHCl}_{3}: \mathrm{MeOH}(8: 2)$ [4x] fornecendo os compostos $4(37,0 \mathrm{mg})$ e $5(68,0 \mathrm{mg})$. A fração metanólica foi submetida ao mesmo procedimento cromatográfico que para fração $n$-butanólica. Foram coletadas 125 frações de $50 \mathrm{~mL}$ cada, sendo que as frações $1 / 56$ foram filtradas em Sephadex LH-20 fornecendo $6(50,0 \mathrm{mg})$ e uma mistura de carboidratos (Figura 1).

\section{Atividade anti-helmíntica}

130 camundongos albinos SW jovens, de ambos os sexos foram mantidos em gaiolas coletivas de polipropileno, nas quais, a limitação das medidas de higiene possibilitou o nível desejado da infecção natural pelo oxiurídeo $A$. tetraptera. Para o teste cada camundongo foi colocado individualmente em uma gaiola. Foram utilizados lotes de 10 camundongos, machos ou fêmeas com peso compreendido entre 20 e 22 g. As gaiolas eram dotadas de piso telado, com o objetivo de facilitar a coleta diária de fezes (Amorim et al., 1987; Amorim \& Borba, 1990). Foram utilizadas doses de 12,0<smiles>[R4]c1cc([R1])c2c(=O)c(-c3ccc([R2])c([R3])c3)coc2c1</smiles>

1: $\mathrm{R}_{1}=\mathrm{R}_{4}=\mathrm{OH}, \mathrm{R}_{2}=\mathrm{OCH}_{3}, \mathrm{R}_{3}=\mathrm{H}$

2: $\mathrm{R}_{1}=\mathrm{H}, \mathrm{R}_{2}=\mathrm{OCH}_{3}, \mathrm{R}_{3}=\mathrm{R}_{4}=\mathrm{OH}$

3: $\mathrm{R}_{1}=\mathrm{R}_{2}=\mathrm{OH}, \mathrm{R}_{3}=\mathrm{H}, \mathrm{R}_{4}=\mathrm{OGlu}$

4: $\mathrm{R}_{1}=\mathrm{OH}, \mathrm{R}_{2}=\mathrm{OCH}_{3}, \mathrm{R}_{3}=\mathrm{H}, \mathrm{R} 4=\mathrm{Oglu}-6 "-1 "$ "'-rha

5: $\mathrm{R}_{1}=\mathrm{OH}, \mathrm{R}_{2}=\mathrm{OCH}_{3}, \mathrm{R}_{3}=\mathrm{H}, \mathrm{R}_{4}=\mathrm{Oglu}-6 "-1 "$ "-apio-5"'-1"'-apio<smiles>Oc1cc(O)c2c(c1)O[C@H](c1ccc(O)c(O)c1)C(O)C2</smiles>

6

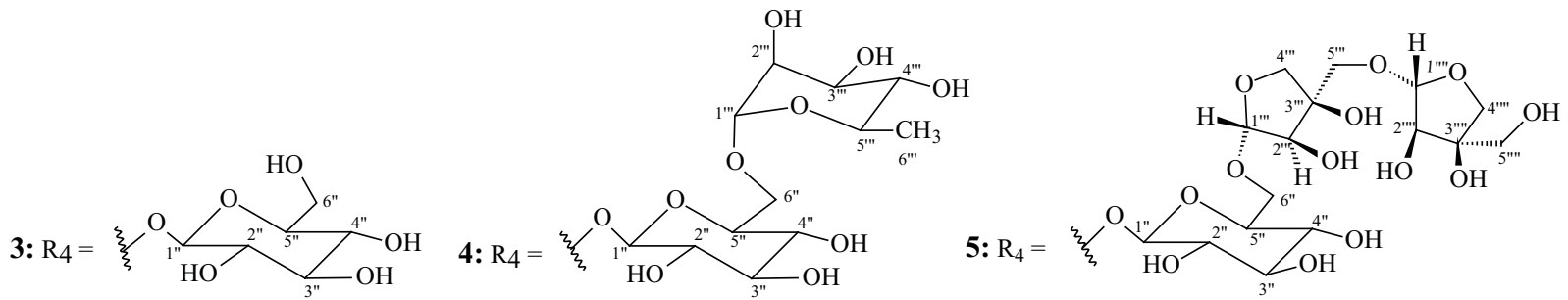

Figura 1. Constituintes químicos de Andira anthelmia. 


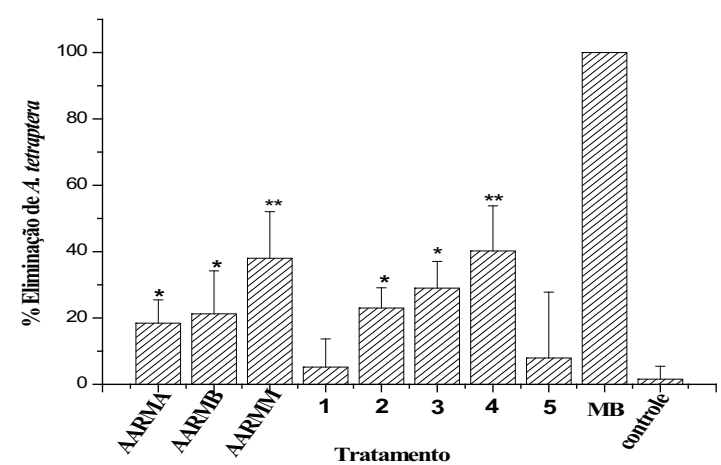

Figura 2. Atividade antihelmíntica em camundongos tratados com as frações e substâncias isoladas de $A$. anthelmia em comparação com o mebendazol (MB) e com animais não tratados (controle). Valores expressos em média \pm DP de nematóides eliminados nos diferentes grupos de animais testados. $n=10$. Os símbolos* e $* *$ indicam valores de $p<0,05$ e $p<0,01$, respectivamente.

e 24,0 mg. $\mathrm{kg}^{-1}$ para os resíduos acetato de etila, $n$-butanol e metanol e doses de $2,0 \mathrm{mg} \cdot \mathrm{Kg}^{-1}$ para as substâncias $\mathbf{1}$, 2, 3, 4, 5 e 6. Os resíduos e substâncias isoladas foram aplicadas por via intragástrica, no volume de 0,04 $\mathrm{mL} . \mathrm{g}^{-1}$, com emprego de sonda de polietileno, durante três dias consecutivos a cada camundongo dos lotes experimentais. As fezes de cada gaiola foram coletadas diariamente, processadas em tamis de malha $125 \mu \mathrm{m}$ e examinadas sob microscópio estereomicroscópio Wild $\mathrm{M}-5$, visando à contagem de oxiurídeos eliminados do $2^{\circ}$ ao 5 o dia, perfazendo um total de quatro exames fecais. Dois lotes de 10 camundongos serviram como controle, sendo que um deles recebeu doses de mebendazol (MB) (Pantelmin ${ }^{\circledR}$, Janssen-Cilag) em três dias consecutivos (2,0 mg. $\left.\mathrm{kg}^{-1}\right)$ e o outro recebeu apenas o veículo $(0,04$ $\left.\mathrm{mL} \cdot \mathrm{g}^{-1}\right)$, objetivando avaliar a eliminação espontânea do helminto. No quinto e último dia dos testes, os animais foram eutanasiados por inalação de vapores de éter etílico e necropsiados, examinando-se o conteúdo do intestino grosso, de modo a avaliar-se o número de $A$. tetraptera remanescentes (Amorim et al., 1999).

\section{Análise estatística}

No teste de atividade anti-helmíntica, os resultados sofreram transformação angular e foram comparados com valores encontrados no lote controle empregando-se o teste $t$ de "Student". O nivel de significância adotado foi $p<0,05$.

\section{RESULTADOS E DISCUSSÃO}

Silva et al., (2003) em estudo posterior, com o extrato metanólico das raízes de $A$. anthelmia comprovou uma significativa atividade anti-helmíntica com uma porcentagem de eliminação de $63,20 \%$ de $A$. tetraptera. Silva et al., (2003) também verificou que o percentual diário de eliminação de $A$. tetraptera observado com os extratos da raiz de $A$. anthelmia foi similar ao comportamento do mebendazol, onde a ação foi lenta, por um período de quatro dias. O extrato metanólico das raízes de $A$. anthelmia mostrou significativa atividade anti-helmíntica com uma porcentagem de eliminação de $A$. tetraptera $(26,71 \%)$. Portanto, este foi sucessivamente particionado fornecendo as frações acetato de etila, $n$-butanol e metanol que apresentaram $18,45 \%, 25,26 \%$ e $37,98 \%$ de eliminação do oxiurídeo respectivamente quando testadas na dose de $12 \mathrm{mg} \cdot \mathrm{kg}^{-1}$. Os animais tratados com os resíduos na dose de 24 mg. $\mathrm{kg}^{-1}$, se mostraram extremamente irritados. Logo na primeira aplicação foi detectada $60 \%$ de mortalidade entre as cobaias. Essa informação alertou para a

Tabela 1. Influência das frações e substâncias isoladas de $A$. anthelmia na eliminação de $A$. tetraptera.

\begin{tabular}{|c|c|c|c|c|}
\hline \multirow{2}{*}{ Tratamento } & \multirow{2}{*}{$\begin{array}{l}\text { Doses } \\
\text { mg.kg }{ }^{-1}\end{array}$} & \multicolumn{2}{|c|}{ Número de helmintos } & \multirow{2}{*}{ \% de eliminação } \\
\hline & & Exame fecal & Necropsia & \\
\hline AARMA $^{\#}$ & 12 & 114 & 521 & $18,45 \pm 7,01$ \\
\hline $\mathrm{AARMB}^{\#}$ & 12 & 406 & 1607 & $21,26 \pm 12,95$ \\
\hline AARMM $^{\#}$ & 12 & 714 & 1880 & $38,0 \pm 14,04$ \\
\hline 1 & 2 & 103 & 1978 & $5,20 \pm 8,5$ \\
\hline 2 & 2 & 700 & 1741 & $23,0 \pm 6,10$ \\
\hline 3 & 2 & 132 & 633 & $29,0 \pm 8,06$ \\
\hline 4 & 2 & 23 & 95 & $40,21 \pm 13,58$ \\
\hline 5 & 2 & 166 & 2088 & $7,95 \pm 5,85$ \\
\hline MB & 2 & 324 & 0 & 100 \\
\hline Controle & --- & 45 & 2836 & $1,56 \pm 3,90$ \\
\hline
\end{tabular}

\#AARMA - Resíduo acetato de etila das raízes de $A$. anthelmia/AARMB - Resíduo butanol das raízes de $A$. anthelmia/AARMM Resíduo metanólica das raízes de $A$. anthelmia/MB - Mebendazol. 
toxidez dos extratos e frações quando empregados em doses elevadas e permitiu um ajuste da dose para uma concentração de $12 \mathrm{mg} \cdot \mathrm{kg}^{-1}$. As frações acetato de etila, $n$-butanol e metanol, por mostrarem uma atividade antihelmíntica significativa, foram submetidas a técnicas de isolamento e purificação por coluna cromatográfica, coluna de Sephadex LH-20 e CCDP. As substâncias foram identificadas por analises espectroscópicas e comparação com dados da literatura; biochanina $\mathrm{A}(\mathbf{1})$ e biochanina A $\quad 7-O-\beta$-D-apiofuranosil- $(1 \rightarrow 5)-\beta$-D-apiofuranosil$(1 \rightarrow 6)$ - $\beta$-D-glucopiranosídeo (5) (Silva et al., 2000), genisteína (2) (Demuner et al., 2003; César et al., 2007), biochanina A 7-O- $\beta$-D-glucopiranosídeo (3) (Rao et al., 1989), biochanina A 7- $O$ - $\alpha$-L-ramnopiranosil- $(1 \rightarrow 6)-\beta$ D-glucopiranosídeo (4) (Ma et al., 1998) e catequina (6) (Foo \& Karchesy, 1989).

Os resultados mostraram que as substâncias 1, 2 e $\mathbf{6}$ apresentaram percentual de eliminação de $A$. tetraptera de $5,20 \%, 5,60 \%$ e $7,95 \%$, respectivamente, quando comparado com o grupo controle (1,56\%), embora não tenham sido estatisticamente significativos. Esses resultados sugerem que haja um sinergismo entre as moléculas existentes nas frações acetato de etila e metanol que influenciam na ação anti-helmíntica. Porém as substâncias 3, 4 e 5 apresentaram significante atividade anti-helmíntica quando comparado com o grupo controle, apesar de serem menos ativas do que o quimioterápico mebendazol $(100 \%)$. Os percentuais de eliminação para as substâncias 3, 4 e 5 foram de $40,21 \%, 20,85 \%$ e $24,21 \%$, respectivamente, sendo a substância 3 a mais ativa dentro da fração $n$-butanólica. Esses resultados são mostrados na Tabela 1 e Figura 2. Esses dados sugerem que nesta série de flavonóides, a presença de unidades glicosídicas na posição C-7 em detrimento de flavonóides agliconas como 3, 4 e 5 podem ser determinantes para a condição de anti-helmíntico.

Levando em consideração tais resultados, infusões de $A$. anthelmia podem ser indicativas de atividade antiparasitária, pois possui substâncias ativas que confirmam esta suposição com atividades terapêuticas interessantes. Porém, devido à alta toxidez as infusões de $A$. anthelmia devem ser administradas com certa precaução, não há estudos de dose segura para consumo humano sendo necessária uma avaliação toxicológica para seu uso popular como um medicamento vermífugo alternativo.

\section{AGRADECIMENTOS}

Os autores agradecem ao CNPq, CAPES e FAPERJ pelas bolsas concedidas e pelo apoio financeiro para o desenvolvimento dessa pesquisa.

\section{REFERÊNCIAS}

Amorim A, Borba HR, Silva WJ 1987. Ação anti-helmíntica de plantas. Rev Bras Farm 68: 64-70.
Amorim A, Borba HR 1990. Ação anti-helmíntica III. Efeito de extratos aquosos de Punica granatum L. (romã) na eliminação de Vampirolepis nana e de oxiurídeos em camundongos. Rev Bras Farm 71: 85-87.

Amorim A, Borba HR, Carauta JPP, Lopes D, Kaplan MAC 1999. Anthelmintic activity of latex of Ficus species. J Ethnopharmacol 64: 255-258.

César IC, Braga FC, Vianna-Soares CD, Nunan EA, Barbosa TAF, Moreira-Campos LM 2007. Determinação de daidzeína, genisteína e gliciteína em cápsulas de isoflavonas por cromatografia em camada delgada (CCD) e cromatografia líquida de alta eficiência (CLAE). Rev Bras Farmacogn 17: 616-625.

Corrêa M 1984. Dicionário de plantas úteis do Brasil e das exóticas cultivadas. Rio de Janeiro: Imprensa Nacional do Rio de Janeiro.

Demuner AJ, Barbosa LCA, Nascimento JC, Vieira JJ, Santos MA 2003. Isolamento e avaliação da atividade nematicida de constituintes químicos de Mucuna cinerea contra Meloidogyne incognita e Heterodera glycines. Quim Nova 26: 335-339.

Foo LY, Karchesy JJ 1989. Procyanidin dimmers and trimers from Douglas Fir Inner Bark. Phytochemistry 28: 1743-1747.

Matos NF 1979. O gênero Andira Lam. (Leguminosae:Papilionoideae) no Brasil. Acta Amazonica 9: 241-266.

Ma WG, Fukushi Y, Hostettmann K, Tahara S 1998. Isoflavonoid glycosides from Eriosma tuberosum. Phytochemistry 49: 251-254.

Pennington T, Lima HC 1995. Two new species of Andira (Leguminosae) from Brazil and the influence of dispersal in determining their distributions. Kew Bull 50: 557-566.

Rao PS, Asheervadam Y, Khalilulliah MD, Murti VVS 1989. A revised structure for the isoflavone lanceolarin. Phytochemistry 28: 957-958.

Silva BP, Velozo LSM, Parente JP 2000. Biochanin A triglycoside from Andira inermis. Fitoterapia 71: 663-667.

Silva SLC, Borba HR, Bonfim RCB, Carvalho MG, Cavalcanti HL, Barbosa CG 2003. Ação Anti-helmíntica de extratos brutos de Andira anthelmia (Vell.) Macbr. e Andira fraxinifolia Benth., em camundongos naturalmente infectados por Vampirolepsis nana e Aspiculuris tetraptera. Parasitol Latinoam 58: 2329.

Zenner L, Regnault JP 2000. A retrospective study of microbiological and parasitological status of laboratory rodents in France. J Exp Anim Sci 40: 211-222. 